\title{
Retraso de la rama descendente de la onda $T$ en electrocardiogramas sin datos de riesgo aparente de muerte súbita
}

\author{
Cuauhtémoc Acoltzin-Vidal, ${ }^{1}$ Elizabeth Rabling-Arellanos ${ }^{2}$ \\ ${ }^{1}$ Universidad de Colima, Facultad de Medicina; ${ }^{2}$ Clínica Cardiológica Privada; Gabinete de Pruebas Especiales. Colima, México
}

\begin{abstract}
Resumen
Introducción: Se ha identificado que la prolongación de la rama descendente de la onda $T$ del electrocardiograma (ECG) puede determinar riesgo de muerte súbita de origen cardiaco, pero se desconoce su importancia en población general. Objetivo: Proporcionar una herramienta de fácil adquisición y aplicación efectiva para identificar riesgo de muerte súbita en población general. Método: Medimos el índice rdT/jT (la rama descendente de la onda T/el espacio entre el punto $j$ y el fin de la T) en 400 electrocardiogramas (ECG) totalmente normales, 656 con alteraciones que no afectan la repolarización ventricular y 82 con bloqueo de rama. Hicimos transformación $Z$ de las curvas de distribución no paramétrica y calculamos razón $Z$ a datos alejados del valor medio. Resultados: La distribución fue asimétrica, sin diferencia en los tres grupos. La transformación Z mostró valor medio de $30 \pm 7$, 10 que sugiere que $95 \%$ de la población debe tener índice rdT/jT $<0.45$. Conclusión: Resultados del índice rdtjT > 0.44 se sitúan más allá de dos desviaciones estándar, por lo tanto, son anormales y deben ser motivo de estudio especializado del portador en busca de riesgo de muerte.
\end{abstract}

PALABRAS CLAVE: Electrocardiografía. Muerte súbita cardiaca. Síndrome de QT largo.

\begin{abstract}
Introduction: Prolongation of the descending branch of the T-wave in the electrocardiogram (ECG) has been identified to be able to determine the risk for sudden death of cardiac origin, but its importance in the general population is not known. Objective: To provide a tool for easy acquisition and effective application to identify the risk of sudden death in the general population. Method: We measured the dbT/jT index (descending branch of the $T$ wave/space between the $j$ point and the end of T), and it was found to be completely normal in 400 ECGs, 656 had alterations that don't affect ventricular repolarization, and 82 had branch block. We carried out the $Z$ transformation of the nonparametric distribution curves and calculated the $Z$ ratio to data far from the mean value. Results: The distribution was asymmetric, with no difference in the three groups. The $Z$ transformation showed a mean value of $30 \pm 7$, which suggests that $95 \%$ of the population has a dbT/jT index $<0.45$. Conclusion: $d b T / j T$ index results $>0.44$ are beyond two standard deviations and are therefore abnormal, which should prompt specialized assessment in order to determine if there is risk for death in the carrier.
\end{abstract}

KEY WORDS: Electrocardiography. Cardiac sudden death. Long QT syndrome.

Correspondencia:

Cuauhtémoc Acoltzin-Vidal

E-mail.com: cuauhtemoc_acoltzin@ucol.mx
Fecha de recepción: 05-08-2017

Fecha de aceptación: 09-08-2017

DOI://dx.doi.org/10.24875/GMM.17002940
Gac Med Mex. 2018;154:198-201

Disponible en PubMed

www.gacetamedicademexico.com 


\section{Introducción}

Ante el impacto que representa morir súbitamente, desde mucho tiempo atrás se ha tratado de identificar a quienes tienen riesgo especial. Con esa intención se han descrito enfermedades como el infarto del miocardio, la estenosis aórtica, la tromboembolia pulmonar, la asfixia por atragantamiento y otras que causan muerte súbita. También se han investigado síndromes electrocardiográficos como el de Lange y Jervell-Nielsen, ${ }^{1}$ el de Romano-Ward, ${ }^{1}$ el de Brugada, ${ }^{2}$ el ahora llamado del punto $\mathrm{J}^{3} \mathrm{y}$ otras entidades que se basan en trastornos del potencial de acción transmembrana como la existencia de potenciales fraccionados, ${ }^{4}$ en especial los potenciales tardíos ventriculares. ${ }^{4,5}$ De primordial importancia son los trastornos de repolarización relacionados con malfuncionamiento de canales iónicos situados en la membrana celular, incluyendo alargamiento $o$ acortamiento del espacio QT, la deformidad y variabilidad de la onda $\mathrm{T}^{2} \mathrm{y}$ prolongación de la duración de la rama descendente de esta, que difieren de los potenciales tardíos ventriculares porque se gestan en diferentes momentos de la actividad eléctrica cardiaca. Mientras que los últimos se relacionan con la terminación de la despolarización ventricular, aquellos tienen que ver con trastorno de la repolarización.

Desde hace casi una década ${ }^{6}$ se ha identificado que la prolongación de la rama descendente de la onda $\mathrm{T}$ del electrocardiograma (ECG) pudiera determinar riesgo de muerte súbita de origen cardiaco. Esta anormalidad electrocardiográfica se ha estudiado en enfermedades que pueden producir muerte súbita como QTc largo o QTc corto, ${ }^{8,9}$ pero también en otras entidades como insuficiencia cardiaca, ${ }^{10}$ miocardiopatía arritmógena de ventrículo derecho, ${ }^{11}$ apnea del sueño, ${ }^{12}$ hipertensión arterial ${ }^{13,14} \mathrm{e}$ infarto del miocardio. ${ }^{15,16} \mathrm{Ha}$ quedado demostrada su asociación con fibrilación ventricular y muerte, por lo menos como complicaciones del infarto.

En la literatura, el concepto se ha manejado con distintos nombres T/QT, ${ }^{6} \mathrm{QTe} / \mathrm{RR}$ y $\mathrm{QTa} / \mathrm{RR},{ }^{10}$ Tpeakend, ${ }^{16}$ TpTe, ${ }^{17}$ Tp-e, ${ }^{13}$ TPE, ${ }^{11}$ Tpe, ${ }^{14}$ entre las cuales ha prevalecido la denominación Tpeak-end/QT ${ }^{16}$ o Tpe/ QT.13

Nosotros propusimos excluir la duración del complejo ventricular (QRS) porque los retardos en la conducción intraventricular falsearían el resultado al aumentar la magnitud del denominador, por lo tanto, restamos ese tiempo y dividimos la rama descendente de la onda T entre el segmento jT (medido desde el punto j y hasta el fin de la onda T), a lo que llamamos índice rdT/jT o índice de Acoltzin-Rabling.

\section{Planteamiento del problema}

No ha quedado claro si en índice $\mathrm{rdT} / \mathrm{jT}$ puede ser anormal en población aparentemente sin riesgo de muerte súbita y por eso preguntamos: ¿los electrocardiogramas de personas aparentemente sin riesgo de muerte súbita presentan alteraciones en el índice rdT/ $\mathrm{jT}$ ?

\section{Objetivo de estudio}

Proporcionar una herramienta de fácil adquisición y aplicación efectiva para identificar riesgo de muerte súbita en población general.

\section{Método}

Se incluyen electrocardiogramas tomados en individuos en reposo, en decúbito dorsal, con la cabeza elevada $30^{\circ}$, medidos por una sola persona y con ayuda de lupa, incluyendo ritmo, frecuencia, P-R, QRS, QTc; eje eléctrico de P, QRS y T en el plano frontal; medición de voltaje ventricular con índices de Cabrera, Lewis, Sokolow y Sokolow-Lion; morfología de las ondas P y QRS; características de la repolarización ventricular como segmento ST y onda T. El QTc se calculó con la fórmula de Bazett. Se separaron tres grupos:

- Todos los electrocardiogramas (denominados PG por población general).

- Electrocardiogramas cuyas medidas resultaron totalmente normales (que recibieron el nombre $\mathrm{S}$, por selecto).

- Electrocardiogramas con QRS mayor de $0.10 \mathrm{~s}$ (denominados BR, por bloqueo de rama).

Se midió el índice rdT/jT: duración desde la cima de la onda T hasta la línea isoeléctrica y duración desde el punto j hasta el fin de la $T$ (en línea isoeléctrica) y se hizo la división en cada caso. Se informan como fracciones (Figura 1).

Se graficaron las distribuciones de los datos de los tres grupos y se compararon. Se calcularon medidas de tendencia central y de dispersión de cada grupo.

Se realizó transformación $Z$ (calculando valor medio y desviación estándar) del grupo $S$ para identificar límites de normalidad a dos y a tres desviaciones de la media. 


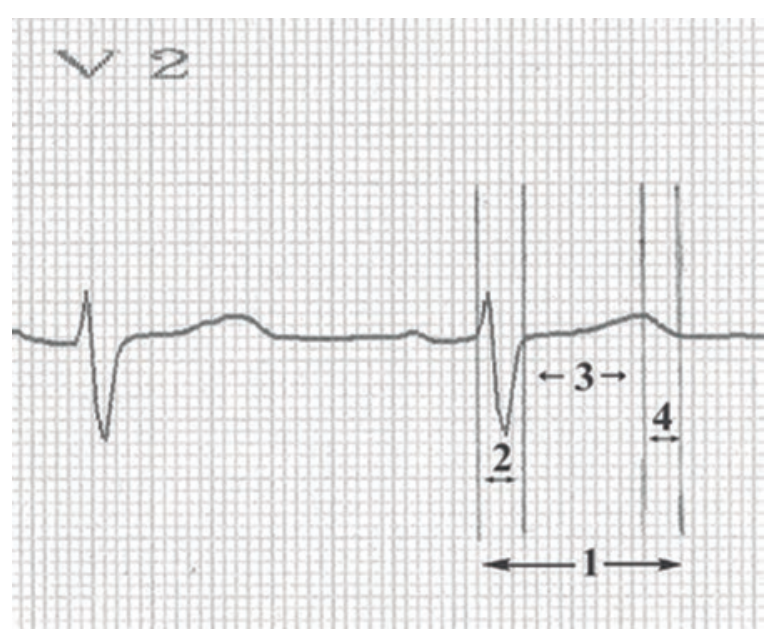

Figura 1. Ejemplo de medición del trazo electrocardiográfico para fines de cálculo del índice rdT/jT. 1) Espacio Q-T. 2) Complejo ventricular o QRS. 3) Espacio J-T. 4) Rama descendente de la onda T.

Se calculó el valor Z para identificar el grado de anormalidad de los datos ubicados afuera de la curva.

\section{Resultados}

Se obtuvieron 656 trazos de la población muestra(PM), 400 selectos (S) y 82 con trastornos de conducción interventricular (BR).

Las distribuciones de los datos mostraron curvas asimétricas desviadas hacia abajo en los tres grupos, con medianas de 29 (PG), 29 (S) y 27 (BR) y modas de 28 (PM), 28(S) y 26 (BR) (Tabla 1), sin diferencia estadísticamente significativa, empleando prueba $U$ entre los grupos.

La transformación $Z$ del grupo $S$ mostró valor medio de $30 \pm 7$ (Tabla 2), sugiriendo que $95 \%$ de la población debe tener índice rdT/jT menor de 0.45 , que puede aceptarse como normal, haciendo inferencia a $99 \%$ de la población si el resultado es hasta de 0.51 .

Al comparar con esta curva normalizada los datos reales identificados por el recorrido intercuartílico del grupo BR, los correspondientes a Q4 se sitúan a 5.14 desviaciones estándar de la media sugerida como poblacional. Llama la atención que los datos situados en Q4 del grupo PM se ubican a 7.85 desviaciones estándar de la curva normal, pero más aún dentro del grupo S, seleccionado porque todos los resultados de medición son normales; también escapan los datos que se sitúan cinco desviaciones estándar.

\section{Discusión}

El cálculo del índice rdT/jT es un instrumento fácil de calcular y verdaderamente útil.
Tabla 1. Distribución normal teórica de los valores del índice rdT/jT en población selecta

\begin{tabular}{ccccccc}
\hline$-3 \mathrm{DE}$ & $-2 \mathrm{DE}$ & $-1 \mathrm{DE}$ & Media & $+1 \mathrm{DE}$ & $+2 \mathrm{DE}$ & $+3 \mathrm{DE}$ \\
0.09 & 0.16 & 0.23 & 0.30 & 0.37 & 0.44 & 0.51 \\
\hline $\mathrm{DE}$, desviación estándar alrededor de la media. Se expresa como fracción
\end{tabular}

Tabla 2. Recorrido intercuartílico de valores del índice $\mathrm{rdT} / \mathrm{jT}$ en diferentes grupos

\begin{tabular}{lcccccc}
\hline & Q0 & Q1 & Mediana & Moda & Q3 & Q4 \\
\hline S & 0.11 & 0.25 & 0.28 & 0.29 & 0.33 & 0.65 \\
PG & 0.06 & 0.25 & 0.28 & 0.29 & 0.35 & 0.85 \\
BR & 0.14 & 0.22 & 0.26 & 0.27 & 0.37 & 0.66 \\
\hline
\end{tabular}

Q, cuartil. Q0 es el dato inferior. Valores expresados como fracciones. S, con

mediciones normales; PG, población general; BR, con bloqueo de rama

Si bien la medición del intervalo QT también es fácil, para estimar su importancia hay que corregir con la fórmula de Bazett que aporta el indicador QTc, que resulta mucho más manejable, si bien los cambios en la duración de QTc pueden ser de varios tipos (por acortamiento o alargamiento) y de estos hay también variedades, además, la duración del intervalo QT cambia por muchos motivos, incluyendo la postura, la temperatura corporal y los medicamentos, ${ }^{18,19}$ lo que le resta confiabilidad.

La identificación del cambio de repolarización típica del síndrome de Brugada se ha modificado de tal modo que si se clasificaba en tres tipos ahora solo se acepta uno y ese también es inconstante. ${ }^{18}$

Los potenciales ventriculares tardíos aparecen en distinto momento de la actividad eléctrica del corazón y aunque han demostrado ser importantes indicadores de arritmias potencialmente mortales posteriores al infarto del miocardio, ${ }^{16}$ son aún más difíciles de identificar: se requieren equipos especiales de electrocardiografía de señal promediada y, en ocasiones, monitoreo ambulatorio ${ }^{20-23}$ disponibles solo en determinados centros. La dificultad aumenta porque algunas veces se observan alteraciones al final de la $\mathrm{R} o$ al principio del segmento ST, que pueden corresponder a la onda épsilon, pero también a ruido. No hay absoluta certidumbre.

En enfoque comparativo se ha informado que la probabilidad de fibrilación ventricular posterior a infarto del miocardio (mortal o no mortal) en personas con potenciales ventriculares tardíos previamente identificados es de $3.4 \%$, y relacionada a estos en población abierta aumenta a $6.7 \%$, lo que señala la existencia de factores de riesgo distintos asociados. ${ }^{5}$ En cambio, la probabilidad de fibrilación ventricular postinfarto asociada con mayor índice "piso-final de T/QT" es de $6 \%{ }^{16}$ 
Los resultados que informamos son teóricos, pues a pesar de haber seleccionado los trazos electrocardiográficos encontramos distribución no paramétrica y tuvimos que transformarla, sin embargo, permiten observar que hay trazos que al calcular el índice $\mathrm{rdT} / \mathrm{j} T$ están tan alejados del valor medio esperado que pudieran tener riesgo especial de fibrilación ventricular y muerte súbita.

Como se ha dicho, se han demostrado los riesgos de arritmia ${ }^{11,16} \mathrm{y}$ de muerte,,${ }^{10,17}$ incluso súbita, ${ }^{10} \mathrm{y}$ se han informado resultados diferentes porque generalmente se han medido cifras absolutas como valores situados sobre el percentil 75 , correspondientes a $>0.20$ para $\mathrm{QTa} / \mathrm{RR}$ ( $\mathrm{Q}$ a la parte alta de T/R-R) $0>0.23$ para QTe/ $R R$ ( $Q$ a fin de $T / R-R$ ) fueron considerados anormales, por asociarse con muerte de cualquier causa o súbita de enfermos con insuficiencia cardiaca; ${ }^{10}$ índice Tp-e/ QT de $0.23 \pm 0.02$ en hipertensos sin descenso nocturno de tensión arterial ${ }^{13}$ o de $0.41 \pm 0.09$ de Tpeak.end/ QT en pacientes con infarto del miocardio y fibrilación ventricular. ${ }^{16}$ Aunque un estudio realizado en perros dio resultado contradictorio, con valores menores de 98.76 ms en índice TpTe de no supervivientes. ${ }^{17}$

El informe de Shenthar et al..$^{16}$ sugiere que el valor máximo aceptable del índice Tpeak.end/QT sea de 0.3 , valor que en la curva de distribución teórica que obtuvimos representa la media. Es más, la cifra esperada a una desviación estándar por encima del valor medio es de $\mathbf{0 . 3 7}$, lo que es absolutamente normal. La explicación posible es que se comparó la duración de la rama descendente de T con QT y las variaciones de duración de QRS podrían cambiar el resultado. No estamos en condición de contestar categóricamente porque nosotros siempre eliminamos la duración del complejo ventricular.

El índice rdT/jT se ve como un indicador accesible de riesgo, con mayor facilidad que los potenciales fraccionados, como los potenciales tardíos que requieren equipo especial de señal fraccionada, y confiable también en comparación con la simple medición del espacio QT.

Proponemos recurrir al índice $\mathrm{rdT} / \mathrm{jT}$ y referirse solo a él para evitar confusión en los informes. Medirlo de manera rutinaria en todos los trazos electrocardiográficos y ampliar el estudio de los casos en que sea mayor de 0.44 ( $45 \%$ de la duración de jT)

\section{Bibliografía}

1. Asensio E, Narváez R, Dorantes J, Oseguera J, Orea A, Hernández P, et al. Conceptos actuales de muerte súbita. Gac Med Mex 2005;141(2):89-97.
2. Nieminen, T, Verrier, RL. Usefulness of T-Wave alternans in sudden death risk stratification and guiding medical therapy. Ann Noninvasive Electrocardiol. 2017;15(3):276-288.

3. Abe A, Ikeda T, Tsukada T, Ishiguro $H$, Miwa $Y$, Miyakoshi M, et al. Circadian variation in late potentials in idiopathic ventricular fibrillation associated with $\mathrm{J}$ waves: insights into alternative pathophysiology and risk stratification. Heart Rhythm 2010;7(5):675-682.

4. Galix. Analizador de potenciales tardíos $L M \circledast$. Manual de operación Disponible en: http://espanol.galix-gbi.com/images/pdf/Manuals/PTV-Manual-ES.pdf

5. Zimmermann M, Sentici A, Adamec R, Metzger J, Mermillod B, Rutishauser W. Long term prognostic significance of ventricular late potential after a first myocardial infarction. Am Heart J. 1997;134(6):1019-1028.

6. Vidal CA, Rabling AE. El índice T/QT señala riesgo de electrocución del corazón. Rev Mex Cardiol. 2006;17(4):170.

7. Acoltzin-Vidal C, Rabling-Arellanos E. En el QTc largo la rama descendente de T mayor de 44\% es anormal. Rev Mex Cardiol. 2014;25(1):3-6.

8. Acoltzin-Vidal C, Rabling-Arellanos E. Comportamiento del índice rdT/ ST en casos de QTc corto comparado con QTc normal. Rev Mex Cardiol. 2008;19(4):208.

9. Chinushi M, Sato A, Izumi D, Furushima H. Nifekalant enlarged the transmural activation-recovery interval difference as well as the peak-toend interval on surface ECG in a patient with short-QT syndrome. J Cardiovasc Electrophysiol.2012;23(8):877-880

10. Cygankiewics I, Zareba W, Vázquez R, Almendral J, Bayes-Genis A, Fiol M, et al. Prognostic value of QT/RR slope in predicting mortality in patients with congestive Heart Failure. J Cardiovasc Electrophisiol. 2008;19(10):1066-1072.

11. Haapalahti $P$, Viitasalo $M$, Kaartinen $M$, Väänänen $H$, Oikarinen $L$, Heliö $\mathrm{T}$, et al. Electrocardiographic ventricular repolarization during cardiovascular autonomic function testing in patients with arrhythmogenic right ventricular cardiomyopathy. Scand Cardiovasc J.2008: 42(6):375-382.

12. Kilicaslan F, Tokatli A, Ozdag F, Uzun M, Uz O, Isilak Z, et al. Tp-e interval, Tp-e/QT ratio, and Tp-e/QTc ratio are prolonged in patients with moderate and severe obstructive sleep apnea. Pacing Clin Electrophysiol.2012;35(8):966-972.

13. Demir M, Uyan U. Evaluation of Tp-e interval and Tp-e/QT ratio in patients with non-dipper hypertension. Clin Exp Hypertens. 2014; 36(5):285-288.

14. Ciobanu A, Gheorghe GS, Ababei M, Deaconu M, llesiu A, Bolohan M, et al. Dispersion of ventricular repolarization in relation to cardiovascular risk factors in hypertension. J Med Life.2014;7(4):545-550.

15. Erikssen G, Liestøl K, Gullestad L, Huagaa KH, Bendz B, Amlie JP. The terminal part of the QT interval (T peak to $\mathrm{T}$ end): a predictor of mortality after acute myocardial infarction. Ann Noninvasive Electrocardiol. 2012;17(2):85-94

16. Shenthar J, Deora S, Rai M, Nanjapa Manjunath C. Prolonged Tpeak-end and Tpeak-end/QT ratio as predictors of malignant ventricular arrhythmias in the acute phase of ST-segment elevation myocardial infarction: a prospective case-control study. Heart Rhythm. 2015; 12(3):484-489.

17. Smetana P, Schmidt A, Zabel M, Hnatkova K, Franz M, Huber K, et al. Assessment of repolarization heterogeneity for prediction of mortality in cardiovascular disease: peak to the end of the T wave interval and nondipolar repolarization components. J Electrocardiol. 2011; 44(3):301-308.

18. Iturralde-Torres P. Arritmias cardiacas. En: Martínez-Ríos MA. Tratado de Cardiología del Instituto de Nacional de Cardiología Ignacio Chávez. México: Intersistemas; 2012.

19. Guill A, Tormos A, Millet J, Roses EJ, Cebrian A, Such-Miquel L, et al. Heterogeneidades inducidas en el intervalo QT mediante enfriamiento/ calentamiento epicárdico local. Estudio experimental. Rev Esp Cardiol. 2014;67(12):993-998.

20. Simson MB. Noninvasive identification of patients at high risk of sudden cardiac death. Signal-averaged electrocardiography. Circulation. 1992;85(Suppl 1):145-151.

21. Reyna CMA, Vildósola RL. Aplicación de Kernels óptimos para mejorar la detección de actividad ventricular tardía. Rev Mex Ing Miomed. 2003:24(1):71-76.

22. Abe A, Kobatashi K, Yuzawa H, Sato H, Fukunaga S, Fugino T, et al. Comparison of late potentials for 24 hours between Brugada syndrome and arrhythmogenic right ventricular cardiomyopathy using a novel signal-averaging system based on Holter ECG. Circ Arrhythm Electrophysiol. 2012;5(4):789-795.

23. Avitia RL, Reyna MA, Bravo-Zanoguera ME, Cetto LA. QRS complex duration enhancement as ventricular late potentials indicator by signal-averaged ECG using time-amplitude alignments. Biomed Tech (Berl). 2013;58(2):179-186 